\title{
Investigating support by minimal detectable displacement in confidence region determination and significance test of displacements
}

\author{
Witold Prószyński ${ }^{1}$. Sławomir Łapiński ${ }^{1}$
}

Received: 22 September 2020 / Accepted: 25 July 2021 / Published online: 15 September 2021

(c) The Author(s) 2021

\begin{abstract}
The Minimal Detectable Displacement (MDD) is an important measure of monitoring networks sensitivity to displacements. In addition to the accuracy criteria, it is used as a detectability criterion in the optimal design of such networks. The paper examines whether the MDD provides grounds for verifying the correctness of the confidence, and the significance thresholds applied in the analyses of the determined displacements. According to our knowledge, the task so formulated has not yet been the subject of research presented in the literature in the field of geodetic determination of displacements. Hence, the approach presented here can be regarded as a new proposal extending the application area of the MDD. The investigations are focused on a probabilistic aspect of combining confidence and detectability as well as significance and detectability by the superimposition of the corresponding ellipsoids and their joint analysis. An initial research result is the diagrams showing a significance index and a non-centrality parameter as functions of the rank of the covariance matrix for displacements and also of system redundancy for specified values of Type I and Type II error probabilities. The diagrams, together with the theoretical basis created within the research, made it possible to analyse and evaluate the support by Minimal Detectable Displacement in confidence region determination and significance test of displacements. Based on the analysis of MDD support, two options of modifying the confidence and significance thresholds related to single point displacements are proposed for practical use.
\end{abstract}

Keywords Ellipsoids superimposition · Error-limit determination · Significance test · MDD support evaluation · Thresholds modification

\section{Introduction}

The transfer to displacement monitoring of the concept of Minimal Detectable Bias (MDB) introduced by Baarda (1968) was made by Pelzer (1972). The term network sensitivity with minimal detectable displacement (MDD) as its measure was introduced and later became an element of network analysis together with accuracy and reliability (Niemeier 1982; Niemeier et al. 1982). The concept of network sensitivity to displacements was elaborated in detail and

Witold Prószyński

witold.proszynski@pw.edu.pl

Sławomir Łapiński

slawomir.lapinski@pw.edu.pl

1 Faculty of Geodesy and Cartography, Warsaw University of Technology, Pl. Politechniki 1, 00-661 Warsaw, Poland extended onto deformation models by Heck (1986). In that paper, the MDD and a sensitivity ellipse for a single network point were first proposed. Some important findings concerning the theory of network sensitivity and datum invariance can be found in, e.g., Even-Tzur (2002, 2010), Xu (1995, 1997). The need for inserting the sensitivity criteria into the network optimization procedures is formulated in (Kuang 1991; Yazji 1997; Alizadeh-Khameneh et al. 2015).

The MDD concept is still commonly used in a priori analyses of monitoring network accuracy and sensitivity. The results of such joint analyses for horizontal networks are presented in the form of a confidence ellipse and a sensitivity ellipse for each network point. However, except for comparing the sizes, no other relationships between these ellipses are considered. Such a limited approach is most certainly due to the lack of a relevant theoretical basis. To our knowledge, in significance tests of computed displacements, the information contained in the sensitivity characteristics has 
not been taken into account as yet. Hence, the need to examine the possibility of using MDD as a source of supporting information both in a priori analyses of monitoring networks accuracy and in significance tests of displacements. If successful, that might extend the range of applications of MDD.

To respond to the above-mentioned need, the objectives of the present paper are the following:

- to create a theoretical basis that would enable one to investigate the extent of MDD support in the analyses of monitoring network accuracy and also in significance tests of the computed displacements,

- to examine the possibility of modifying the above-mentioned procedures so as to use the supporting information contained in the MDD.

It is necessary to emphasize that the paper concerns only the models with parameters being network points displacements. It does not deal with the models containing also strain parameters as used in network deformation analysis.

\section{Brief review of basic concepts and notation}

The review concentrates on minimum-constraint datum definition as commonly used in the monitoring of displacements and the measures of network sensitivity to displacements.

\subsection{Minimum-constraint datum definitions}

Let us consider a displacement monitoring model based on observation differences from two measurement campaigns

$\mathbf{A} \cdot \mathbf{d}=\Delta \mathbf{l}+\mathbf{v}_{\Delta \mathbf{l}}, \quad \mathbf{C}_{\Delta \mathbf{l}}$

$\mathbf{S} \cdot \mathbf{d}=\mathbf{0}$

where $\mathbf{A}(n \times u)$-design matrix, $\operatorname{rank} \mathbf{A}=u-d(d$-network defect $), \mathbf{d}(u \times 1)$-vector of displacements, $\Delta \mathbf{l}(n \times 1)$-vector of observation differences, $\mathbf{v}_{\Delta \mathrm{l}}(n \times 1)$-vector of random errors in $\Delta \mathbf{l}$ (with opposite sign), $\mathbf{C}_{\Delta \mathbf{l}}(n \times n)$-covariance matrix for $\Delta \mathbf{l}$ (positive definite), $\mathbf{S}(d \times u)$-coefficient matrix in datum constraints.

We shall denote by $\mathbf{A}_{\mathrm{s}}$ and $(\Delta \mathbf{l})_{\mathrm{s}}$ the elements of the standardized model (1) corresponding to $\mathbf{A}$ and $\Delta \mathbf{l}$, respectively.

The models as in (1) having point displacements as parameters are basically equivalent to those with point velocities.

The matrix $\mathbf{S}$ has the following properties

$\operatorname{rank}\left[\begin{array}{c}\mathbf{A} \\ \mathbf{S}\end{array}\right]=u, \quad \operatorname{rank} \mathbf{S}=d$
The minimum-constraint datum definition eliminates the network defect without causing observation distortions. There are several types of such constraints, e.g., free net constraints.

Below, we present the least squares (LS) solution for the standardized model (1), distinguishing the following two ways in which the result of the global model test can be used in defining the covariance matrix for displacements

(i) using the a priori variance factor $\sigma_{\mathrm{o}}^{2}=1$, where $\sigma_{\mathrm{o}}^{2}$ is a variance of the standardized observation difference $(\Delta \mathrm{l})_{\mathrm{s}, i}(i=1, \ldots, n)$;

$$
\mathbf{C}_{\hat{\mathbf{d}}}=\sigma_{\mathrm{o}}^{2} \mathbf{Q}_{\hat{\mathbf{d}}}=\mathbf{Q}_{\hat{\mathbf{d}}}
$$

(ii) using the estimator (unbiased) of $\sigma_{\mathrm{o}}^{2}$, i.e., $\hat{\sigma}_{\mathrm{o}}^{2}$, termed the a posteriori variance factor;

$$
\hat{\mathbf{C}}_{\hat{\mathbf{d}}}=\hat{\sigma}_{\mathrm{o}}^{2} \mathbf{Q}_{\hat{\mathbf{d}}}
$$

$\operatorname{Ad}(\mathrm{i}) \hat{\mathbf{d}}=\left(\mathbf{A}_{\mathrm{s}}^{\mathrm{T}} \mathbf{A}_{\mathrm{s}}\right)_{\mathbf{S}}^{-} \mathbf{A}_{\mathrm{s}}^{\mathrm{T}}(\Delta \mathbf{l})_{\mathrm{s}} ; \mathbf{C}_{\hat{\mathbf{d}}}=\left(\mathbf{A}_{\mathrm{s}}^{\mathrm{T}} \mathbf{A}_{\mathrm{s}}\right)_{\mathbf{S}}^{-} \quad \operatorname{rank} \mathbf{C}_{\hat{\mathbf{d}}}=u-d$

$$
\text { for free net constraints } \mathbf{C}_{\hat{\mathbf{d}}}=\left(\mathbf{A}_{\mathrm{s}}^{\mathrm{T}} \mathbf{A}_{\mathrm{s}}\right)_{\mathbf{S}_{\mathrm{o}}}^{-} \equiv\left(\mathbf{A}_{\mathrm{s}}^{\mathrm{T}} \mathbf{A}_{\mathrm{s}}\right)^{+}
$$

Ad (ii) $\hat{\mathbf{d}}=\left(\mathbf{A}_{\mathrm{s}}^{\mathrm{T}} \mathbf{A}_{\mathrm{s}}\right)_{\mathrm{s}}^{-} \mathbf{A}_{\mathrm{s}}^{\mathrm{T}}(\Delta \mathbf{l})_{s} ; \hat{\mathbf{C}}_{\hat{\mathbf{d}}}=\hat{\sigma}_{\mathrm{o}}^{2}\left(\mathbf{A}_{\mathrm{s}}^{\mathrm{T}} \mathbf{A}_{\mathrm{s}}\right)_{\mathrm{s}}^{-}$, rank $\hat{\mathbf{C}}_{\hat{\mathbf{d}}}=\operatorname{rank} \mathbf{C}_{\hat{\mathbf{d}}}$

In the above formulas, $\left(\mathbf{A}_{\mathrm{s}}^{\mathrm{T}} \mathbf{A}_{\mathrm{s}}\right)_{\mathrm{S}}^{-}$is a reflexive $g$-inverse of $\mathbf{A}_{\mathrm{s}}^{\mathrm{T}} \mathbf{A}_{\mathrm{s}}$, such that $\mathbf{S} \cdot\left(\mathbf{A}_{\mathrm{s}}^{\mathrm{T}} \mathbf{A}_{\mathrm{s}}\right)_{\mathbf{S}}^{-}=\mathbf{0},\left(\mathbf{A}_{\mathrm{s}}^{\mathrm{T}} \mathbf{A}_{\mathrm{s}}\right)^{+}$is a pseudoinverse.

Also known is another datum definition, termed overconstraint datum (where $\mathbf{S}(w \times u)$, rank $\mathbf{S}=w, w>d$ ), fixing some network points. It eliminates the network defect but causes observation distortions. Its use is bounded by the restrictions on mutual displacements of the points being fixed and the types of measured quantities.

\subsection{Network sensitivity to displacements}

The measures of sensitivity to displacements are derived on the basis of hypotheses testing

$H_{\mathrm{o}}: \mathrm{E}(\hat{\mathbf{d}})=\mathbf{0}$

$H_{\mathrm{a}}: \mathrm{E}(\hat{\mathbf{d}}) \neq \mathbf{0}$

where $\hat{\mathbf{d}}$ is the LS estimator of the vector of displacements as in (5) and (6).

If the covariance matrix $\mathbf{C}_{\hat{\mathbf{d}}}$ as in (3) is used, the test statistic is 
$\Phi=\hat{\mathbf{d}}^{\mathrm{T}} \mathbf{C}_{\hat{\mathbf{d}}}^{+} \hat{\mathbf{d}}$

- under $H_{0}$, $\Phi$ has a central $\chi_{\mathrm{h}}^{2}$ distribution, $h$ is a rank of $\mathbf{C}_{\hat{\mathrm{d}}}$,

- under $H_{\mathrm{a}}, \Phi$ has a non-central $\chi_{\mathrm{h}}^{2}$ distribution, with a noncentrality parameter

$$
\lambda=\hat{\mathbf{d}}^{\mathrm{T}} \mathbf{C}_{\mathbf{d}}^{+} \hat{\mathbf{d}}
$$

A vector $\hat{\mathbf{d}}$ is considered detectable if $\lambda>\lambda_{\mathrm{h}, \boldsymbol{\alpha}_{\mathrm{o}}, \beta_{\mathrm{o}}}$, where $\lambda_{\mathrm{h}, \alpha_{\mathrm{o}}, \beta_{\mathrm{o}}}$ is a critical value of non-centrality parameter determined for the specified values of Type I and Type II error probabilities $\alpha_{o}$ and $\beta_{o}$.

If the covariance matrix $\hat{\mathbf{C}}_{\hat{\mathbf{d}}}$ as in (4) is used, the test statistic is

$\Phi_{\mathrm{F}}=\frac{\hat{\mathbf{d}}^{\mathrm{T}} \mathbf{Q}_{\hat{\mathbf{d}}}^{+} \hat{\mathbf{d}}}{h \cdot \hat{\sigma}_{\mathrm{o}}^{2}}$

where $\mathbf{Q}_{\hat{\mathrm{d}}}$ is a cofactor matrix, $h$ is a rank of $\mathbf{Q}_{\hat{\mathrm{d}}}, \hat{\sigma}_{\mathrm{o}}^{2}$ is the a posteriori variance factor

- under $H_{\mathrm{o}}, \Phi_{\mathrm{F}}$ has a central $F_{\mathrm{h}, \mathrm{r}}$ distribution ( $\mathrm{r}$ is a model redundancy; $\mathrm{r}=n-u+d$ ),

- under $H_{\mathrm{a}}, \Phi_{\mathrm{F}}$ has a non-central $F_{\mathrm{h}, \mathrm{r}}$ distribution, with a noncentrality parameter

$\lambda_{\mathrm{F}}=\frac{\hat{\mathbf{d}}^{\mathrm{T}} \mathbf{Q}_{\hat{\mathbf{d}}}^{+} \hat{\mathbf{d}}}{\hat{\sigma}_{\mathrm{o}}^{2}}$

A critical value of the non-centrality parameter is denoted here by $\lambda_{\mathrm{h}, \mathrm{r}, \alpha_{0}, \beta_{\mathrm{o}}}$. A vector $\hat{\mathbf{d}}$ is considered detectable if $\lambda_{\mathrm{F}}>\lambda_{\mathrm{h}, \mathrm{r}, \alpha_{\mathrm{o}}, \beta_{\mathrm{o}}}$, where $\alpha_{\mathrm{o}}$ and $\beta_{\mathrm{o}}$ as above.

For a single point vector with a non-singular covariance matrix, $h$ is a number of the vector components.

On the basis of the formulas (8) and (10), we can write the equations of $h$-dimensional sensitivity ellipsoids for the case of $\mathbf{C}_{\hat{\mathbf{d}}}$ and $\hat{\mathbf{C}}_{\hat{\mathbf{d}}}$, respectively, i.e.,

$\mathbf{d}^{\mathrm{T}} \mathbf{C}_{\hat{\mathbf{d}}}^{+} \mathbf{d}=\lambda_{\mathrm{h}, \alpha_{\mathrm{o}}, \beta_{\mathrm{o}}}$

$\frac{\mathbf{d}^{\mathrm{T}} \mathbf{Q}_{\mathbf{d}}^{+} \mathbf{d}}{\hat{\sigma}_{\mathrm{o}}^{2}}=\lambda_{\mathrm{h}, \mathrm{r}, \mathrm{\alpha}_{\mathrm{o}}, \beta_{\mathrm{o}}}$

Based on (11) and (12), we get the commonly used sensitivity measure as Minimal Detectable Displacement (MDD), correspondingly for the case of $\mathbf{C}_{\hat{\mathbf{d}}}$ and $\hat{\mathbf{C}}_{\hat{\mathbf{d}}}$ (Niemeier 1982, 1985) $\|\mathbf{d}\|_{\min }=\sqrt{\frac{\lambda_{\mathrm{h}, \alpha_{o}, \beta_{\mathrm{o}}}}{\Lambda_{\max }}}\|\mathbf{d}\|_{\min }=\hat{\sigma}_{\mathrm{o}} \sqrt{\frac{\lambda_{\mathrm{h}, \mathrm{r}, \alpha_{\mathrm{o}}, \beta_{\mathrm{o}}}}{\Lambda_{\max }}}$

where $\Lambda_{\max }$ is a maximal eigenvalue of the matrix $\mathbf{C}_{\hat{\mathbf{d}}}^{+}$and $\mathbf{Q}_{\hat{\mathbf{d}}}^{+}, \hat{\sigma}_{\mathrm{o}}$ is a square root of the a posteriori variance factor.

According to (Hsu and Hsiao 2002), both the minimal and the maximal value of MDD should be taken into consideration. The maximal value is based on a minimal eigenvalue $\Lambda_{\text {min }}$.

Datum invariance of the sensitivity quadratic form as in (7) is an important issue in the subject of the paper since the MDD defined on its basis is applied in different types of datum definition. It is known that the form is invariant to the choice of the type of minimum-constraint datum definition. A concise proof, complementary to the existing proofs (e.g., Even-Tzur 2010), is presented in "Appendix A" section. It covers all possible types of datum definition.

A non-centrality parameter for a test statistic (10) in the case of the model (1) with weights of observation differences is considered in "Appendix B" section.

\section{Investigating MDD support in accuracy analysis and in the testing of displacements}

In investigations, we operate with $h$-dimensional displacement vectors $(h \geq 1)$. For simplicity, the corresponding regions of confidence, significance and sensitivity, being line segments $(h=1)$, ellipses $(h=2)$, ellipsoids $(h=3)$ and hyper-ellipsoids $(h>3)$, will be termed for all $h$ the ellipsoids, e.g., (Pope 2008).

The proposal covers the following two task cases:

- Case I Confidence region determination supported by network sensitivity characteristics (variance factor option (i))

- Case II Significance test of computed displacements supported by network sensitivity characteristics (variance factor option (i) and (ii)).

As a final result, the extent of the support is evaluated.

\subsection{Creating of the theoretical basis}

\subsubsection{Variance factor option (i)}

A property of the test statistic $\Phi=\hat{\mathbf{d}}^{\mathrm{T}} \mathbf{C}_{\hat{\mathbf{d}}}^{+} \hat{\mathbf{d}}$ under $H_{\mathrm{o}}$ and $H_{\mathrm{a}}$, i.e., $\Phi=\lambda$ for $h \geq 1$ (see (7), (8)), as expressed in terms of the corresponding values of $\Phi$ and $\lambda$ (i.e., $u$ and $\lambda$, respectively), means the following equality 
$u_{\mathrm{h}, \alpha}=\lambda_{\mathrm{h}, \alpha, \beta}$

For a given $\alpha$, we get $\beta$ from the above equality. We will say that such $\beta$ is coordinated with a given $\alpha$. Assuming an arbitrary value of $\beta$ not coordinated with a given $\alpha$ and denoted by $\tilde{\beta}$ will result in $u_{\mathrm{h}, \alpha} \neq \lambda_{\mathrm{h}, \alpha, \tilde{\beta} \text {. }}$

Let critical values of $\Phi$ and $\lambda$ be denoted by $u_{\mathrm{o}}$ and $\lambda_{\mathrm{o}}$, where $u_{\mathrm{o}}=u_{\mathrm{h}, \alpha_{\mathrm{o}}}, \lambda_{\mathrm{o}}=\lambda_{\mathrm{h}, \alpha_{0}, \beta_{\mathrm{o}}} ; \alpha_{\mathrm{o}}$ and $\beta_{\mathrm{o}}$ are the values of $\alpha$ and $\beta$ assumed in $u_{\mathrm{o}}$ and $\lambda_{\mathrm{o}}$, respectively. Putting, e.g., $\alpha_{\mathrm{o}}=0.05$ and $\beta_{\mathrm{o}}=0.20$, we get from (14) $u_{\mathrm{o}} \neq \lambda_{\mathrm{o}}$, and hence, we can see that $\beta_{0}=0.20$ is not coordinated with $\alpha_{\mathrm{o}}=0.05$. The value of $\beta$ coordinated with $\alpha_{\mathrm{o}}=0.05$ will be denoted by $\bar{\beta}$.

The investigation of MDD support requires knowledge on the relationship between the sizes and probability features of the following three concentric and similar $h$-dimensonal ellipsoids, such as sensitivity ellipsoid $\left(E_{\text {sen }}\right)$, confidence ellipsoid $\left(E_{\text {con }}\right)$ (also termed as a limiterror ellipsoid) and significance ellipsoid $\left(E_{\mathrm{sig}}\right)$.

Let us consider equations of these ellipsoids, referring to the expression for a test statistic $\Phi$ as in (7). The components of the vector $\mathbf{d}$ will be treated here as algebraic variables.

$E_{\text {sig }} \quad \mathbf{d}^{\mathrm{T}} \mathbf{C}_{\hat{\mathbf{d}}}^{+} \mathbf{d}=u_{\mathrm{o}} ; u_{\mathrm{o}}=u_{\mathrm{h}, \alpha_{\mathrm{o}}} ; \alpha_{\mathrm{o}}$ - significance level

$u_{\mathrm{o}}$ - a critical value of the variable $\Phi$ such that $P\left\{\Phi>u_{\mathrm{h}, \alpha_{\mathrm{o}}}\right\}=\alpha_{\mathrm{o}}$

$E_{\text {con }} \quad \mathbf{d}^{\mathrm{T}} \mathbf{C}_{\hat{\mathbf{d}}}^{+} \mathbf{d}=u_{\mathrm{o}(\gamma)} ; u_{\mathrm{o}(\gamma)}=u_{\mathrm{h}, \gamma_{\mathrm{o}}} ; \gamma_{\mathrm{o}}$-confidence level $u_{\mathrm{o}(\gamma)}$ - a critical value of the variable $\Phi$ such that $P\left\{\Phi<u_{\mathrm{h}, \gamma_{\mathrm{o}}}\right\}=\gamma_{\mathrm{o}}$

$E_{\text {sen }} \mathbf{d}^{\mathrm{T}} \mathbf{C}_{\hat{\mathbf{d}}}^{+} \mathbf{d}=\lambda_{\mathrm{o}} ; \lambda_{\mathrm{o}}=\lambda_{\mathrm{h}, \alpha_{\mathrm{o}}, \beta_{\mathrm{o}}}(\operatorname{see}(11))$

$\lambda_{\mathrm{o}}$ - a critical value of a non-centrality parameter of the variable $\Phi$.

$E_{\text {sen }}$ represents a detectability threshold for displacements. It is easy to prove that every vector that satisfies Eq. (11) is an MDD in this direction.

Putting $\gamma_{\mathrm{o}}=1-\alpha_{\mathrm{o}}$ in (16), we get $u_{\mathrm{h}, \gamma_{\mathrm{o}}}=u_{\mathrm{h}, \alpha_{\mathrm{o}}}$ which reduces the number of sizes to be analyzed to two, the corresponding parameters being $u_{\mathrm{h}, \alpha_{\mathrm{o}}}$ and $\lambda_{\mathrm{h}, \alpha_{0}, \beta_{\mathrm{o}}}$.

The values of $u_{\mathrm{h}, \alpha_{\mathrm{o}}}$ were computed iteratively on the basis of the arguments of cumulative distribution function (CDF) for a $\chi_{\mathrm{h}}^{2}$-distributed random variable. For the computation of $\lambda_{\mathrm{h}, \alpha_{\mathrm{o}}, \beta_{\mathrm{o}}}$ use was made of the efficient and sufficiently accurate numerical program developed by Aydin and Demirel (2005) according to the algorithm and the associated theory given in (Gaida and Koch 1985). The results of the computation are presented in Table 1 and Fig. 1.

The symbol $h_{\alpha_{0}, \beta_{\mathrm{o}}}^{*}$ in Table 1 denotes a crossing point of the curves $u_{\mathrm{o}}(h)$ and $\lambda_{\mathrm{o}}(h)$ (see Fig. 1).

We can see in Fig. 1 that with the increase in $h$, both the curves increase monotonically, the former one at a higher rate.

At a certain value of $h$, denoted as $h_{\alpha_{0}, \beta_{0}}^{*}$ (being equal to 7.3), these curves cross each other. For $h<h_{\alpha_{0}, \beta_{0}}^{*}$, we have $u_{\mathrm{o}}<\lambda_{\mathrm{o}}$, whereas for $h>h_{\alpha_{0}, \beta_{\mathrm{o}}}^{*}$ we have $u_{\mathrm{o}}>\lambda_{\mathrm{o}}$. For a given $h$, the symbol $\bar{\beta}$ denotes the value of $\beta$ coordinated with $\alpha_{\mathrm{o}}=0.05$. For $u_{\mathrm{o}}<\lambda_{\mathrm{o}}$, we have $\bar{\beta}>\beta_{\mathrm{o}}$ and for $u_{\mathrm{o}}>\lambda_{\mathrm{o}}, \bar{\beta}<\beta_{\mathrm{o}}$.
Table 1 Values of $u_{\mathrm{o}}$ and $\lambda_{\mathrm{o}}$ for different $h\left(\alpha_{0}=0.05, \beta_{0}=0.20\right)$; $h_{\alpha_{0}, \beta_{0}}^{*}=7.3$

Fig. 1 Parameters $u_{0}$ and $\lambda_{0}$ as functions of $h ; \alpha_{0}=0.05$, $\beta_{\mathrm{o}}=0.20$

\begin{tabular}{|c|c|c|c|c|c|c|c|c|c|c|c|c|}
\hline$h$ & 1 & 2 & 3 & 4 & 5 & 6 & 7 & 8 & 9 & 10 & 20 & 50 \\
\hline$u_{\mathrm{o}}$ & 3.8 & 6.0 & 7.8 & 9.5 & 11.1 & 12.6 & 14.1 & 15.5 & 16.9 & 18.3 & 31.4 & 67.5 \\
\hline$\lambda_{\mathrm{o}}$ & 7.8 & 9.6 & 10.9 & 11.9 & 12.8 & 13.6 & 14.4 & 15.0 & 15.6 & 16.2 & 21.0 & 30.2 \\
\hline
\end{tabular}

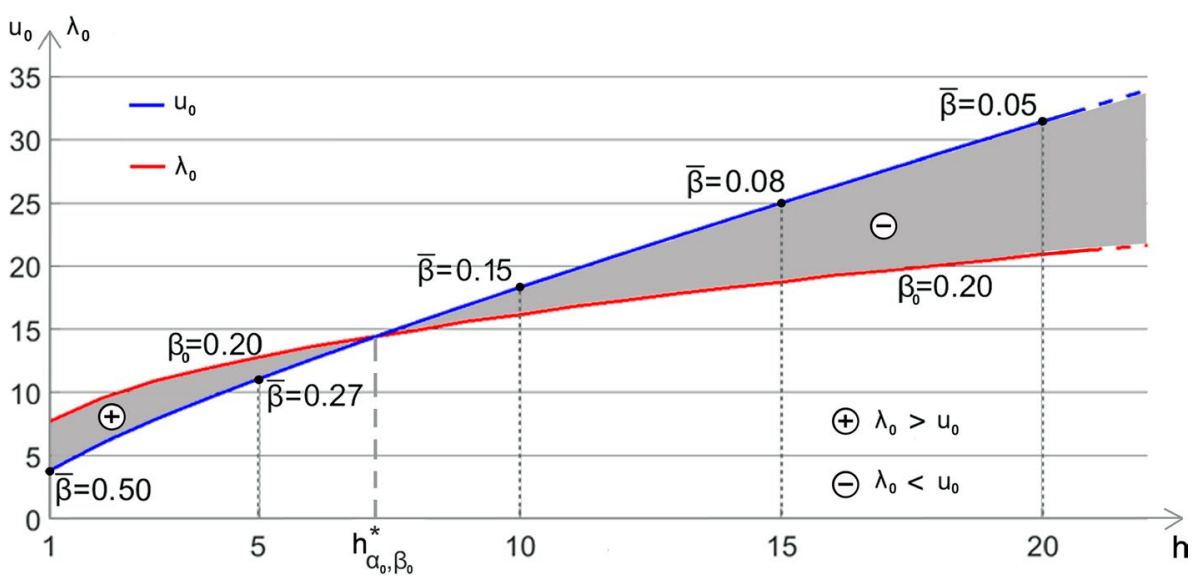


We can also notice that the values of $\bar{\beta}$ decrease with the increase in $h$, which is consistent with the findings in (Aydin 2011) focused on the power of the test.

The properties as presented above for $\alpha_{\mathrm{o}}=0.05, \beta_{\mathrm{o}}=0.20$ can be observed for other values of $\alpha_{\mathrm{o}}$ and $\beta_{\mathrm{o}}$.

For single network points, we have $h<h_{\alpha_{0}, \beta_{0}}^{*}$, so the size of $E_{\text {sen }}$ is greater than that of $E_{\text {sig }}$ and $\mathrm{E}_{\text {con }}\left(\right.$ with $\left.\gamma_{\mathrm{o}}=1-\alpha_{\mathrm{o}}\right)$. For networks taken as a whole or for the subsets of their points, such that $h>h_{\alpha_{0}, \beta_{0}}^{*}$, we have the opposite situation.

\subsubsection{Variance factor option (ii)}

From the formulas (9) and (10), it follows that the property analogous to that in the variance factor option (i), i.e., $\Phi=\lambda$, does not hold directly for $\Phi_{\mathrm{F}}$. We can get this effect by introducing a rescaled variable $\Phi_{\mathrm{F}}$, defined by

$\Psi=h \cdot \Phi_{\mathrm{F}}$

Hence,

$\Psi=\frac{\hat{\mathbf{d}}^{\mathrm{T}} \mathbf{Q}_{\hat{\mathbf{d}}}^{+} \hat{\mathbf{d}}}{\hat{\sigma}_{0}^{2}}$

The values of $\Psi$ will be denoted by " $w$ ".

The variable does not have an identifiable probability distribution but has a property useful for the present derivations, i.e.,

Property If $r \rightarrow \infty$, the probability distribution of the variable $\Psi \rightarrow \chi_{\mathrm{h}}^{2}$ (the property is well known in mathematical statistics).

So, since with $r \rightarrow \infty \quad \hat{\sigma}_{\mathrm{o}}^{2} \rightarrow \sigma_{\mathrm{o}}^{2}$, the variable $\Psi \rightarrow \frac{\hat{\mathbf{d}}^{\mathrm{T}} \mathbf{Q}_{\hat{d}}^{+} \hat{\mathbf{d}}}{\sigma_{0}^{2}}=\hat{\mathbf{d}}^{\mathrm{T}} \mathbf{C}_{\hat{\mathbf{d}}}^{+} \hat{\mathbf{d}} \equiv \Phi$.

As was assumed in Sect. 2.1, $\sigma_{\mathrm{o}}^{2}=1$.

Now, we find the critical value of $\Phi$ for a given significance level $\alpha$. Denoting the values of $\Phi_{\mathrm{F}}$ by “ $z$ ", we shall write

$P\left\{\Phi_{\mathrm{F}}>z_{\mathrm{h}, \mathrm{r}, \alpha}\right\}=\alpha$
Substituting (17) and duly modifying the internal inequality, we obtain finally

$P\left\{\Psi>w_{\mathrm{h}, \mathrm{r}, \alpha}\right\}=\alpha$

where $w_{\mathrm{h}, \mathrm{r}, \alpha}=h \cdot z_{\mathrm{h}, \mathrm{r}, \alpha}$.

The variable $\Psi$, being compatible with $\Phi$ as regards properties and scale, satisfies the equality $\Psi=\lambda_{\mathrm{F}}$ for $h \geq$ 1 and $r \geq 1$. So, the values of $\Psi$ and $\lambda_{\mathrm{F}}$, i.e., $w$ and $\lambda_{\mathrm{F}}$ are bound by the equality

$w_{\mathrm{h}, \mathrm{r}, \alpha}=\lambda_{\mathrm{h}, \mathrm{r}, \alpha, \beta}$

In analogy to (14), $\beta$ is coordinated with a given value of $\alpha$.

Let critical values of $\Psi$ and $\lambda_{\mathrm{F}}$ be denoted by $w_{\mathrm{O}}$ and $\lambda_{\mathrm{F}, 0}$, where $w_{\mathrm{o}}=w_{\mathrm{h}, \mathrm{r}, \alpha_{\mathrm{o}}}, \lambda_{\mathrm{F}, \mathrm{o}}=\lambda_{\mathrm{h}, \mathrm{r}, \alpha_{\mathrm{o}}, \beta_{\mathrm{o}}}\left(\alpha_{\mathrm{o}}=0.05, \beta_{\mathrm{o}}=0.20\right)$. We may check that $w_{\mathrm{o}} \neq \lambda_{\mathrm{F}, \mathrm{o}}$, and hence, $\beta_{\mathrm{o}}=0.20$ is not coordinated with $\alpha_{0}=0.05$. The value of $\beta$ coordinated with $\alpha_{\mathrm{o}}=0.05$ will be denoted by $\bar{\beta}$ (for simplicity of notation, the same symbol as for variance factor option (i) is used).

For Case II (variance factor option (ii)), we need to acquire knowledge on the relationship between the sizes of the corresponding significance ellipsoid and the sensitivity ellipsoid. We shall denote these ellipsoids by $\hat{E}_{\mathrm{sig}}$ and $\hat{E}_{\mathrm{sen}}$, respectively. We write their equations without additional explanations

$\hat{E}_{\text {sig }} \frac{\mathbf{d}^{\mathrm{T}} \mathbf{Q}_{\hat{\mathbf{d}}}^{+} \mathbf{d}}{\hat{\sigma}_{\mathrm{o}}^{2}}=w_{\mathrm{o}} ; \quad w_{\mathrm{o}}=w_{\mathrm{h}, \mathrm{r}, \mathbf{\alpha}_{\mathrm{o}}}$

$\hat{E}_{\mathrm{sen}} \frac{\mathbf{d}^{\mathrm{T}} \mathbf{Q}_{\mathbf{d}}^{+} \mathbf{d}}{\hat{\sigma}_{\mathrm{o}}^{2}}=\lambda_{\mathrm{F}, \mathrm{o}} ; \lambda_{\mathrm{F}, \mathrm{o}}=\lambda_{\mathrm{h}, \mathrm{r}, \mathrm{\alpha}_{\mathrm{o}}, \beta_{\mathrm{o}}}$

To the above-mentioned property of $\Psi$ concerned with $r$ increasing to infinity, we may add the following

- since with $r \rightarrow \infty w_{\mathrm{h}, \mathrm{r}, \alpha} \rightarrow u_{\mathrm{h}, \alpha}$ and $\lambda_{\mathrm{h}, \mathrm{r}, \alpha, \beta} \rightarrow \lambda_{\mathrm{h}, \alpha, \beta}$, so $\hat{E}_{\text {sig }} \rightarrow E_{\text {sig }}$ and $\hat{E}_{\text {sen }} \rightarrow E_{\text {sen }}$.
Table 2 Values of $w_{\mathrm{o}}$ and $\lambda_{\mathrm{F}, \mathrm{o}}$ for different $h$ and $r\left(\alpha_{0}=0.05\right.$, $\left.\beta_{\mathrm{o}}=0.20\right) ; h_{\alpha_{0}, \beta_{0}, \mathrm{r}}^{*}$

\begin{tabular}{|c|c|c|c|c|c|c|c|c|c|c|c|c|c|c|}
\hline$r$ & $h$ & 1 & 2 & 3 & 4 & 5 & 6 & 7 & 8 & 9 & 10 & 20 & 50 & $h_{\alpha_{0}, \beta_{0}, r}^{*}$ \\
\hline & $w_{\mathrm{o}}$ & 6.6 & 11.6 & 16.2 & 20.8 & 3 & 29.7 & 34.1 & 38.5 & 43.0 & 47.4 & 91.2 & 222.2 & $\mathrm{x}$ \\
\hline & $\lambda_{\mathrm{F}, \mathrm{o}}$ & 12.3 & 18.7 & 24.5 & 30.1 & 35.7 & 41.2 & 46.7 & 52.1 & 57.5 & 62.9 & 116.8 & 277.9 & \\
\hline & $w_{\mathrm{o}}$ & 5.0 & 8.2 & 11.1 & 13.9 & 16.6 & 19.3 & 21.9 & 24.6 & 27.2 & 29.8 & 55.5 & 31.9 & 49.9 \\
\hline & $\lambda_{\mathrm{F}, \mathrm{o}}$ & 9.7 & 13.2 & 16.2 & 19.0 & 21.7 & 24.3 & 26.9 & 29.5 & 32.0 & 34.5 & 59.1 & 131.8 & \\
\hline & $w_{\mathrm{o}}$ & 4.4 & .0 & 3 & 1 & 13.6 & 15.6 & 17.6 & 19.6 & 21.5 & 23.5 & 42.5 & 8.3 & 12.0 \\
\hline & $\lambda_{\mathrm{F}, \mathrm{o}}$ & 8.7 & 11.2 & 13.3 & 15.1 & 16.8 & 18.4 & 20.0 & 21.5 & 23.0 & 24.4 & 38.4 & 78.4 & \\
\hline \multirow[t]{2}{*}{100} & $w_{\mathrm{o}}$ & 3.9 & 6.2 & 8.1 & 9.9 & 11.5 & 13.1 & 14.7 & 16.3 & 17.8 & 19.3 & 33.5 & 73.9 & 7.9 \\
\hline & $\lambda_{\mathrm{F}, \mathrm{o}}$ & 8.0 & 9.9 & 11.3 & 12.5 & 13.6 & 14.5 & 15.4 & 16.2 & 17.0 & 17.8 & 24.3 & 40.1 & \\
\hline
\end{tabular}

$\mathrm{x}$-value difficult to determine 
Fig. 2 Parameters $w_{\mathrm{o}}$ and $\lambda_{\mathrm{F}, \mathrm{O}}$ as functions of $h$, for $r=10$ and $r=50\left(\alpha_{\mathrm{o}}=0.05, \beta_{\mathrm{o}}=0.20\right)$

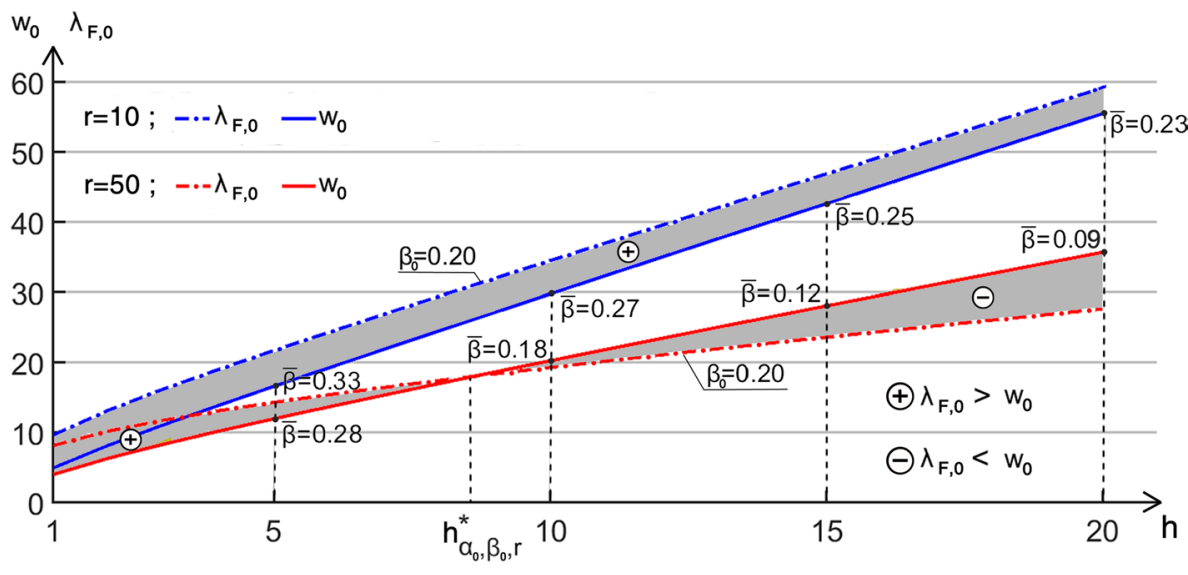

The values $w_{\mathrm{o}}$ were obtained by rescaling the values $z_{\mathrm{h}, \mathrm{r}, \alpha_{\mathrm{o}}}$, computed iteratively based on the arguments of CDF for $F_{\mathrm{h}, \mathrm{r}}$-distributed variable. Like the values of $\lambda_{\mathrm{o}}$ in option (i), the values of $\lambda_{\mathrm{F}, \mathrm{o}}$ were computed with the use of a numerical program developed by Aydin (2011). The results of the computation are presented in Table 2 and Fig. 2.

The symbol $h_{\alpha_{0}, \beta_{0}, \mathrm{r}}^{*}$ in Table 2 denotes a crossing point of the curves $w_{\mathrm{o}}(h)$ and $\lambda_{\mathrm{F}, \mathrm{o}}(h)$ for some $r$ (see Fig. 2).

Figure 2 presents the curves $w_{\mathrm{o}}(h)$ and $\lambda_{\mathrm{F}, \mathrm{o}}(h)$ for $r=10$ and $r=50$. The curves are generally similar to their equivalents in Fig. 1. We can see in Table 2 and Fig. 2 that the values of $h$ where these curves intersect (denoted here as $\left.h_{\alpha_{0}, \beta_{0}, \mathrm{r}}^{*}\right)$ increase with the decrease in $r$. For $r=10$, the intersection of the curves falls outside the diagram.

Interpretation of the cases $\mathrm{h}<h_{\alpha_{0}, \beta_{0}, \mathrm{r}}^{*}$ and $h>h_{\alpha_{0}, \beta_{0}, \mathrm{r}}^{*}$ is like that given for option (i). The values of $\bar{\beta}$ decrease with the increase in $h$.

Fig. 3 Presentation of ellipsoids for analysis in Case I (1, 2, 3 -vectors of characteristic location, $\gamma_{\mathrm{o}}=1-\alpha_{\mathrm{o}}$ )

\subsection{Analysis for Case I}

\subsubsection{Variance factor option (i)}

Usually, both the confidence ellipse and the sensitivity ellipse are presented together as a result of network quality analysis. We provide the description of characteristic displacement vectors and some probabilistic features of each of the ellipses (here-ellipsoids).

The values of $\bar{\beta}$ in Fig. 3 are computed for $h=2$ $\left(h<h_{\alpha_{0}, \beta_{0}}^{*}\right), h=15\left(h>h_{\alpha_{0}, \beta_{0}}^{*}\right)$.

Since we consider the a priori accuracy analysis, the vectors 1, 2, 3 represent potential (not determined) displacement vectors.

Deterministic description of the characteristic vectors:

\begin{tabular}{lc}
\hline for $h<h_{\alpha_{0}, \beta_{\mathrm{o}}}^{*}$ & for $h>h_{\alpha_{0}, \beta_{\mathrm{o}}}^{*}$ \\
\hline $\begin{array}{l}\text { 1. error-affected displacements } \\
\text { displ. under detectability } \\
\text { threshold }\end{array}$ & $\begin{array}{l}\text { 1. displ. under detectability } \\
\text { threshold }\end{array}$ \\
$\begin{array}{l}\text { 2. error-free displacements } \\
\text { displ. under detectability } \\
\text { threshold }\end{array}$ & $\begin{array}{c}\text { 2. detectable displacements } \\
\text { error-affected displacements }\end{array}$ \\
$\begin{array}{l}\text { 3. error-free displacements } \\
\text { detectable displacements }\end{array}$ & $\begin{array}{l}\text { 3. error-free displacements } \\
\text { detectable displacements }\end{array}$ \\
\hline
\end{tabular}

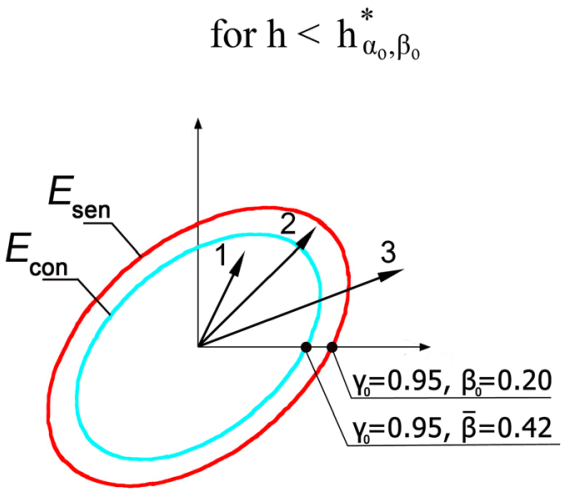

for $h>h_{\alpha_{0}, \beta_{0}}^{*}$

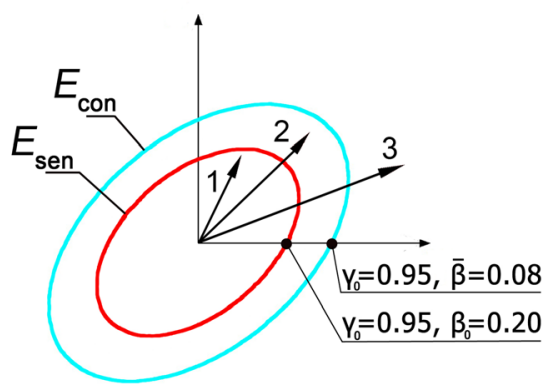


For a zone between the ellipsoids (see vector 2), we observe some inconsistency in the above description. It is due to the fact that the confidence related and the sensitivity related qualifications concern different assumptions (i.e., hypothesis $H_{\mathrm{o}}$ and hypothesis $H_{\mathrm{a}}$ ), with Type I and Type II error probabilities not mutually coordinated. The detectability threshold $\lambda_{\mathrm{o}}$ is different from the error-limit $u_{\mathrm{o}(\gamma)}$.

\subsection{Analysis for Case II}

\subsubsection{Variance factor option (i)}

For the analysis of significance combined with sensitivity, we present the significance ellipsoid and the sensitivity ellipsoid together with the description of characteristic displacement vectors and some probabilistic features of each of the ellipsoids.

The values of $\bar{\beta}$ in Fig. 4 are computed for $h=2$ $\left(h<h_{\alpha_{0}, \beta_{0}}^{*}\right), h=15\left(h>h_{\alpha_{0}, \beta_{0}}^{*}\right)$.
The deterministic description is as follows

\begin{tabular}{ll}
\hline for $h<h_{\alpha_{0}, \beta_{0}}^{*}$ & for $h>h_{\alpha_{0}, \beta_{0}}^{*}$ \\
\hline 1. insignificant displacement & 1. displ. under detectability threshold \\
$\begin{array}{l}\text { 2. significant displacement } \\
\text { displ. under detectability }\end{array}$ & $\begin{array}{c}\text { 2. detectable displacement } \\
\text { insignificant displacement }\end{array}$ \\
$\begin{array}{l}\text { 3. significant displacement } \\
\text { detectable displacement }\end{array}$ & $\begin{array}{c}\text { 3. detectable displacement } \\
\text { significant displacement }\end{array}$ \\
\hline
\end{tabular}

Like in Case I, for a zone between the ellipsoids (see vector 2), we observe some inconsistency in the above description. The cause of inconsistency is as that explained in Case I. Here, the detectability threshold $\lambda_{\mathrm{o}}$ is different from the significance threshold $u_{\mathrm{o}}$.

We may readily classify the computed $h$-dimensional displacement vector $\hat{\mathbf{d}}$ either as 1, 2 or 3, respectively, by means of the following checks based on the values $u$ of the test statistic $\Phi$, i.e.,

for $h<h_{\alpha_{0}, \beta_{0}}^{*}: u \leq u_{\mathrm{o}}, u_{\mathrm{o}}<u \leq \lambda_{\mathrm{o}}, u>\lambda_{\mathrm{o}}$

for $h>h_{\alpha_{0}, \beta_{\mathrm{o}}}^{*}: u \leq \lambda_{\mathrm{o}}, \lambda_{\mathrm{o}}<u \leq u_{\mathrm{o}}, u>u_{\mathrm{o}}$
Fig. 4 Presentation of ellipsoids for analysis in Case II, option (i) $(1,2,3$-vectors of characteristic location)
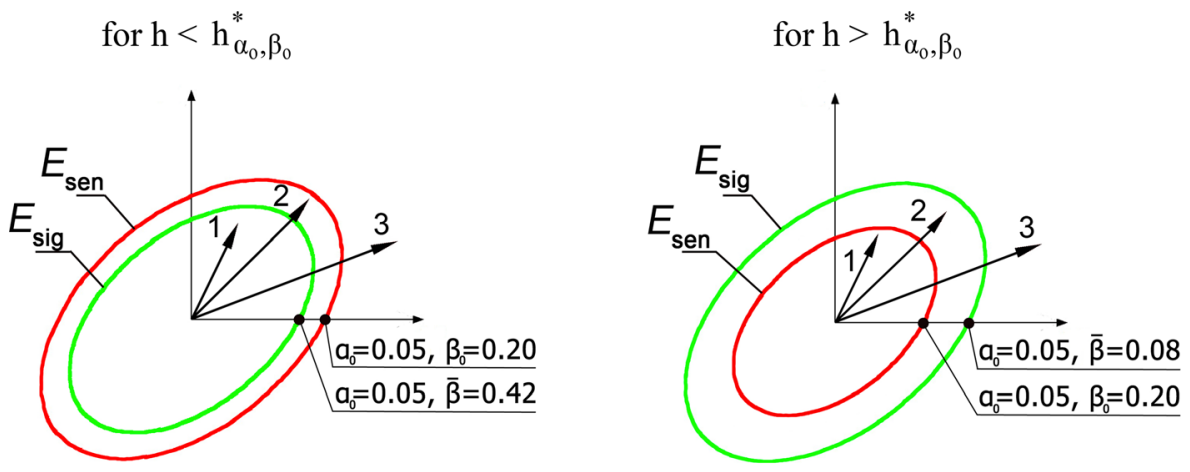

Fig. 5 Presentation of ellipsoids for analysis in Case II, option (ii) $(1,2,3$-vectors of characteristic location)
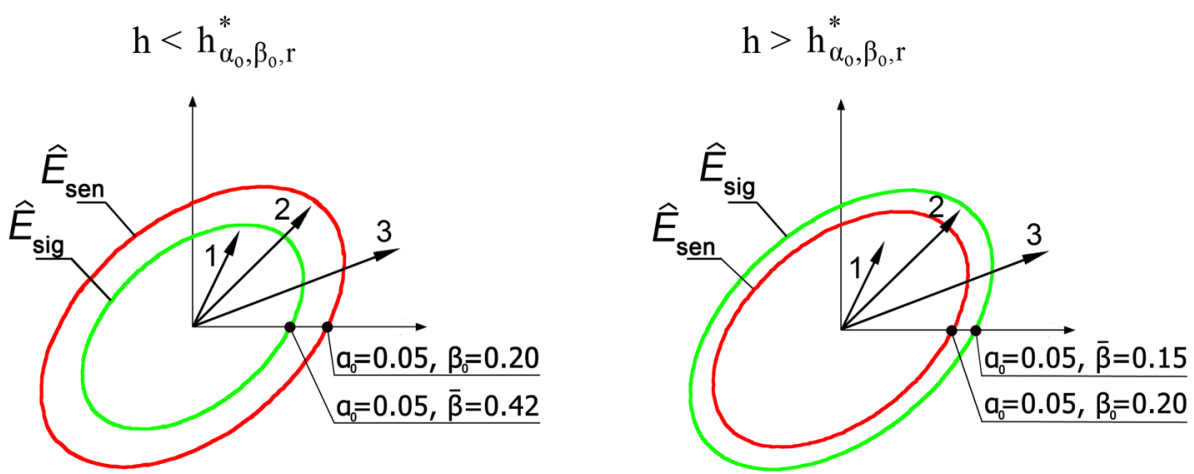


\subsubsection{Variance factor option (ii)}

Since the deterministic description of the displacement vectors is analogous to that in option (i), we only present both the ellipsoids together with their probabilistic features (Fig. 5).

To classify the computed $h$-dimensional displacement vector $\mathbf{d}$ either as 1,2 or 3 , respectively, we use here the following checks based on the values $\mathrm{w}$ of the variable $\Psi$ and $\lambda_{\mathrm{F}}$ as in (21) and (22)

for $h<h_{\alpha_{0}, \beta_{0}, r}^{*}: w \leq w_{\mathrm{o}}, w_{\mathrm{o}}<w \leq \lambda_{\mathrm{F}, \mathrm{o}}, w>\lambda_{\mathrm{F}, \mathrm{o}}$

for $h>h_{\alpha_{0}, \beta_{0}, \mathrm{r}}^{*}: w \leq \lambda_{\mathrm{F}, \mathrm{o}}, w_{\mathrm{f}, \mathrm{r}, \lambda}<w<w_{\mathrm{f}, \mathrm{r}, \mathrm{\alpha}}, w>w_{\mathrm{o}}$

For this variance factor option, the greater the system redundancy, the lower is the detectability threshold, and the smaller are the displacement vectors that can be considered as insignificant. It is therefore recommended to always ensure an appropriately high redundancy level.

The values of $\bar{\beta}$ in Fig. 5 are computed for $r=20$ and $h=2\left(h<h_{\alpha_{0}, \beta_{0}, \mathrm{r}}^{*}\right), h=20\left(h>h_{\alpha_{0}, \beta_{0}, \mathrm{r}}^{*}\right)$.

\subsection{Evaluating the MDD support in task Cases I and II}

The MDD support is understood here as providing grounds for verifying the correctness of the significance (or confidence) threshold. The support is the greater the closer is the sensitivity ellipsoid to significance (or confidence) ellipsoid.

The value of $\bar{\beta}$ obtained from the equality (14) with $\alpha_{\mathrm{o}}=0.05$ (or $\gamma_{\mathrm{o}}=1-\alpha_{\mathrm{o}}$ ) depends except $h$ only on the value of $\alpha_{\mathrm{o}}$. Hence, $\bar{\beta}$ is only partially connected with the detectability threshold $\lambda_{\mathrm{o}}$, which is defined on both $\alpha_{\mathrm{o}}$ and $\beta_{\mathrm{o}}$. The difference $\left|\lambda_{\mathrm{o}}-u_{\mathrm{o}}\right| \neq 0$ is a main cause of inconsistency in the description of vector qualification discussed in Sect. 3.2 (Fig. 3) and Sect. 3.3 (Figs. 4 and 5). The smaller is $\bar{\beta}$, and the smaller is $\left|\lambda_{\mathrm{o}}-u_{\mathrm{o}}\right|$, the greater is MDD support. The analysis of the above-mentioned relationships led to introducing of the following directlyproportional measure of MDD support, defined by

for $h<h_{\alpha_{0}, \beta_{\mathrm{o}}}^{*} \quad Q\left(\underline{\beta}, \frac{u_{\mathrm{o}}}{\lambda_{\mathrm{o}}}\right)$

for $h>h_{\alpha_{0}, \beta_{\mathrm{o}}}^{*} Q\left(\underline{\beta}, \frac{\lambda_{\mathrm{o}}}{u_{\mathrm{o}}}\right)$
Table 3 Values of $Q$ for some $h\left(\alpha_{\mathrm{o}}=0.05, \beta_{\mathrm{o}}=0.20\right)$; italics concerns $h>h_{\alpha_{0}, \beta_{\mathrm{o}}}^{*}$

\begin{tabular}{lllllllllllllll}
\hline$h$ & 1 & 2 & 3 & 4 & 5 & 6 & 7 & 8 & 9 & 10 & 20 & 30 & 40 & 50 \\
\hline$\underline{\beta}$ & 0.50 & 0.58 & 0.64 & 0.69 & 0.73 & 0.76 & 0.79 & 0.81 & 0.84 & 0.86 & 0.96 & 0.98 & 0.995 & 0.998 \\
$\frac{u_{0}}{\lambda_{0}} ; \frac{\lambda_{0}}{u_{0}}$ & 0.49 & 0.62 & 0.72 & 0.79 & 0.86 & 0.92 & 0.98 & 0.97 & 0.92 & 0.89 & 0.67 & 0.56 & 0.49 & 0.45 \\
\hline
\end{tabular}

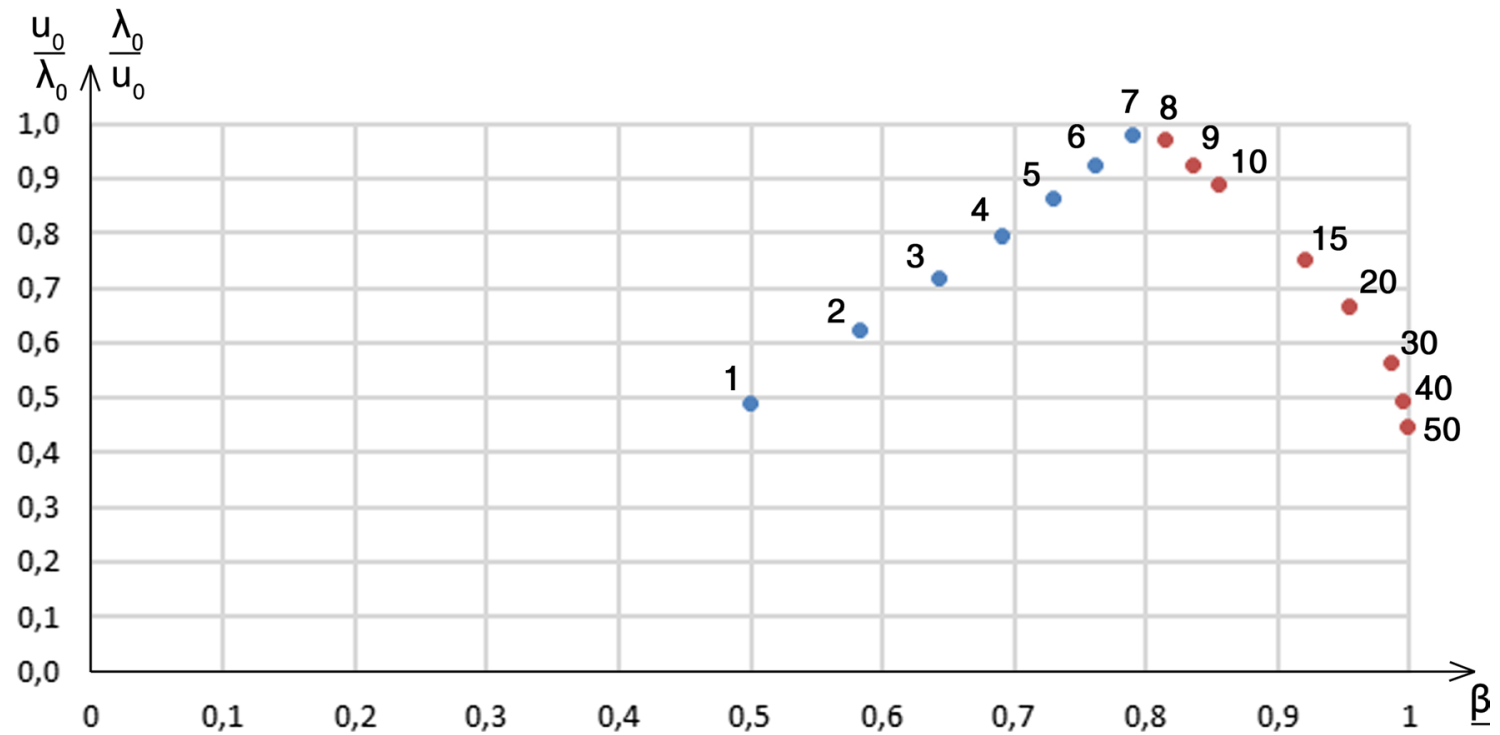

Fig. 6 Plot of $Q$ values for some $h\left(\alpha_{\mathrm{o}}=0.05, \beta_{\mathrm{o}}=0.20\right) ; Q$ points are labeled with the values of $h ; h<h_{\alpha_{0}, \beta_{\mathrm{o}}}^{*}$ in blue, $h>h_{\alpha_{0}, \beta_{\mathrm{o}}}^{*}$ in red 
where $\underline{\beta}=1-\bar{\beta}$ is a power of the test for $u_{\mathrm{o}}=\lambda_{\mathrm{o}}$, the second components in $Q$ are equal to 1 for $h=h_{\alpha_{0}, \beta_{0}}^{*}$.

Since both the parameters in $Q$ are mutually incomparable, in the case of opposite trends in their functions of $h$, ranking of $Q$ will not be possible. Therefore, the ranking of the MDD support will then have to be carried out with respect to each of the parameters separately.

The measure of MDD support as in (27) and (28) is constructed on the basis of parameters used in Table 1 and diagrams in Fig. 1. Hence, variability of $Q$ values is some other representation of the above-mentioned diagrams.

The values of $Q$ for some $h$ are presented in Table 3 and Fig. 6.

The results in Table 3 and Fig. 6 apply to Task Case I and Task Case II (variance factor option (i)). We may conclude that the MDD support is the smallest for single point displacements in $1 \mathrm{D}$ networks $\left(\underline{\beta}=0.5, \frac{u_{\mathrm{o}}}{\lambda_{\mathrm{o}}}=0.49\right)$ and becomes successively greater for $2 \mathrm{D}\left(\underline{\beta}=0.58, \frac{u_{\mathrm{o}}}{\lambda_{\mathrm{o}}}=0.62\right)$ and $3 \mathrm{D}$ networks $\left(\underline{\beta}=0.64, \frac{u_{0}}{\lambda_{0}}=0.72\right)$. For $h>h_{\alpha_{0}, \beta_{0}}^{*}$ (i.e., for global network displacements), in terms of $\beta$ the support is increasing, whereas in terms of $\frac{\lambda_{0}}{u_{0}}$ it is decreasing. For $h=50, \underline{\beta}$ is approaching 1 while $\frac{u_{\mathrm{o}}}{\lambda_{\mathrm{o}}}$ falls down to 0.45 .
In analogy to the definition in (27) and (28), the measure of MDD support for Task Case II (variance factor option (ii)) is defined as follows

for $h<h_{\alpha_{0}, \beta_{0}, \mathrm{r}}^{*} \quad Q\left(\underline{\beta}, \frac{w_{\mathrm{o}}}{\lambda_{\mathrm{F}, \mathrm{o}}}\right)$

for $h>h_{\alpha_{0}, \beta_{0}, \mathrm{r}}^{*} Q\left(\underline{\beta}, \frac{\lambda_{\mathrm{F}, \mathrm{o}}}{w_{\mathrm{o}}}\right)$

where $\beta=1-\bar{\beta}$ is a power of the test for $\lambda_{\mathrm{F}}=w_{\mathrm{o}}, \bar{\beta}$ is obtaine $\bar{d}$ from the equality (20), i.e., $w_{\mathrm{h}, \mathrm{r}, \alpha_{\mathrm{o}}}=\lambda_{\mathrm{h}, \mathrm{r}, \alpha_{\mathrm{\alpha}}, \bar{\beta}}$, the second components in $Q$ are equal to 1 for $h=h_{\alpha_{0}, \beta_{0}, \mathrm{r}}^{*}$. The MDD support is presented in Table 4 and Fig. 7.

Like in Fig. 6, the MDD support is the smallest for single point displacements in 1D networks and becomes successively greater for 2D and 3D networks. And also, for $h>h_{\alpha, \beta, r}^{*}$ (i.e., for global network displacements), in terms of $\beta$ the support is increasing, whereas in terms of $\frac{\lambda_{0}}{u_{0}} \overline{i t}$ is decreasing.

According to the property presented in Sect. 3.1 (variance factor option (ii)), with the increase in $r$, the MDD support becomes closer and closer to that in Fig. 6 for Task Case II (variance factor option (i)).
Table 4 Values of $Q$ for some $h$ and $r=20\left(\alpha_{\mathrm{o}}=0.05, \beta_{\mathrm{o}}=0.20\right)$; italics concerns $h>h_{\alpha_{0}, \beta_{0}, \mathrm{r}}^{*}$

\begin{tabular}{lllllllllllllll}
\hline$h$ & 1 & 2 & 3 & 4 & 5 & 6 & 7 & 8 & 9 & 10 & 20 & 30 & 40 & 50 \\
\hline$\beta$ & 0.51 & 0.58 & 0.63 & 0.67 & 0.70 & 0.72 & 0.74 & 0.76 & 0.77 & 0.78 & 0.85 & 0.88 & 0.89 & 0.90 \\
$\frac{w_{\mathrm{o}}}{\lambda_{\mathrm{E} 0}} ; \frac{\lambda_{\mathrm{F}, 0}}{w_{\mathrm{o}}}$ & 0.50 & 0.62 & 0.70 & 0.76 & 0.81 & 0.85 & 0.88 & 0.91 & 0.94 & 0.96 & 0.90 & 0.85 & 0.82 & 0.80 \\
\hline
\end{tabular}

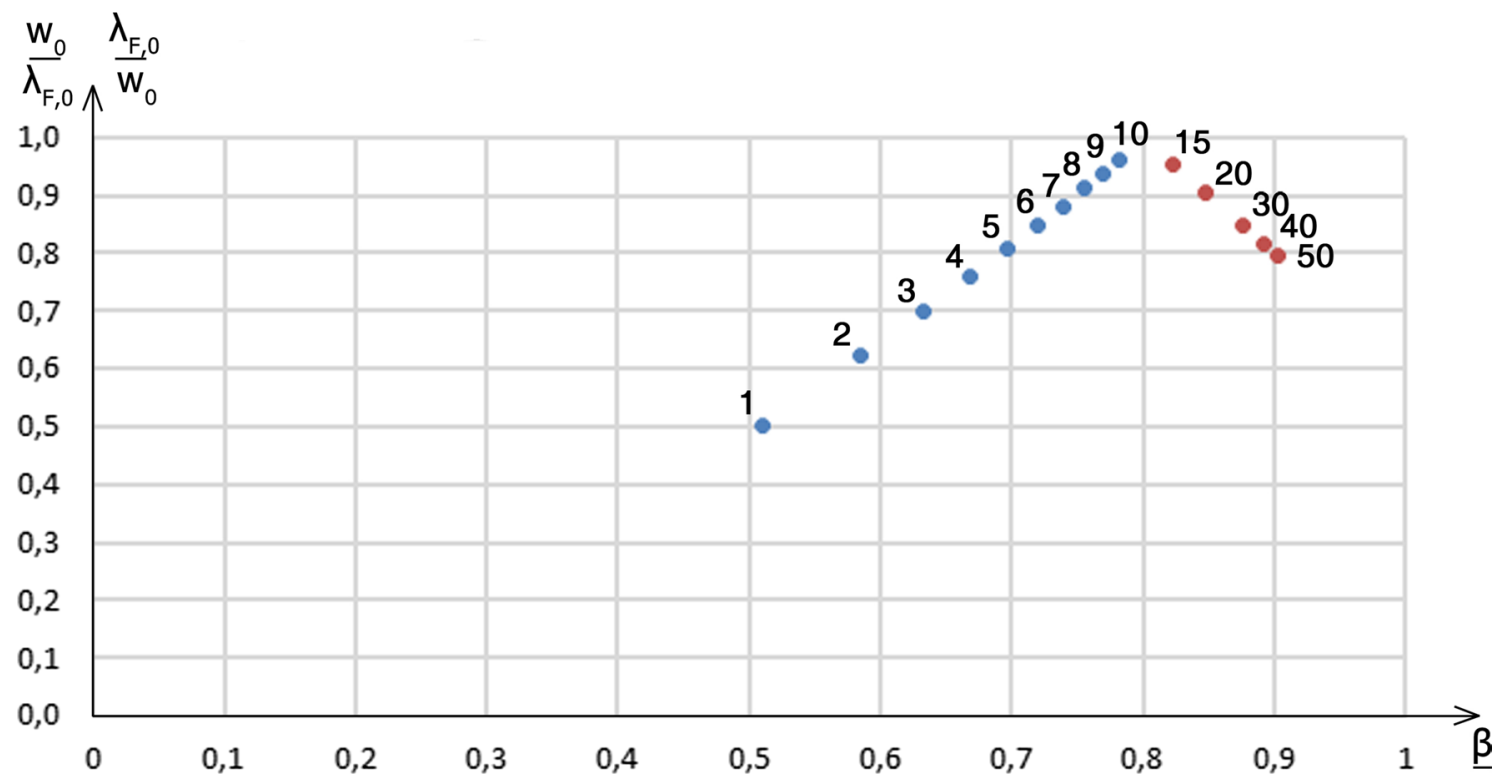

Fig. 7 Plot of $Q$ values for some $h$ and $r=20\left(\alpha_{\mathrm{o}}=0.05, \beta_{\mathrm{o}}=0.20\right) ; Q$ points are labeled with the values of $h ; h<h_{\alpha_{0}, \beta_{0}, \mathrm{r}}^{*}$ in blue, $h>h_{\alpha_{0}, \beta_{0}, \mathrm{r}}^{*}$ in red 
The diagrams in Figs. 6 and 7 confirm the theory in Sect. 3.1 in that the MDD support for both the variance factor options increases up to the intersection point $\left(h_{\alpha_{0}, \beta_{o}}^{*}\right.$ or $\left.h_{\alpha_{0}, \beta_{0}, \mathrm{r}}^{*}\right)$, and behind this point it increases in terms of the first parameter but decreases in terms of the second parameter. Since the smaller is $r$ (variance factor option (ii)), the intersection point falls for greater $h$, a zone of the increasing MDD support becomes longer. This effect was observed on a diagram for $r=10$ where $h_{\alpha_{0}, \beta_{0}, \mathrm{r}}^{*}=49.9$, not shown in the present paper.

\subsection{Investigating the possibility of thresholds modification}

The question now arises whether the MDD support gives grounds for advantageous modification of the thresholds in the significance test of displacements (or confidence region determination) and detectability for $h=1,2,3$. The following two possibilities can be taken into account:

- lowering the detectability threshold while keeping the sensitivity (or confidence) threshold fixed, i.e., $E_{\text {sen }}$ is brought toward $E_{\text {sig }}$ in terms of size,

- raising the sensitivity (or confidence) threshold while keeping the detectability threshold fixed, i.e., $E_{\text {sig }}$ is brought toward $E_{\text {sen }}$ in terms of size.

From the analyses carried out for the first possibility, it follows that such a modification would result in an unacceptable increase in the probability of the Type II error. Maximum values of this probability, expressed in terms of the power of the test $\beta$ are presented in Table 3 and Fig. 4. So, we pass to the second possibility.

Below, we present the analysis for two reasonable options of modifying the significance threshold for both the variance options, i.e., (i) and (ii). In Option 1, we assume $u_{\mathrm{o}, \mathrm{m}}=$ $0.5\left(u_{\mathrm{o}}+\lambda_{\mathrm{o}}\right)$, while in Option $2 u_{\mathrm{o}, \mathrm{m}}=\lambda_{\mathrm{o}}$, where the subscript "m" denotes the modified quantity. In Option $1, E_{\mathrm{sig}, \mathrm{m}}$ lies in between $E_{\text {sig }}$ and $E_{\text {sen }}$, while in Option $2 E_{\text {sig,m }} \equiv E_{\text {sen }}$.

The data for the analysis are presented in Tables 5 and 6. The symbol $\bar{\beta}_{m}$ denotes $\beta$ coordinated with $\alpha_{\mathrm{o}, \mathrm{m}}$, and as in Sect. 3.1 (variance factor option (i)), the symbol $\bar{\beta}$ denotes $\beta$ coordinated with $\alpha_{\mathrm{o}}$ (here $\alpha_{\mathrm{o}}=0.05$ ).

Ad option 1 (variance factor option (i))

For $h<h_{\alpha_{0}, \beta_{0}}^{*}$, the Type I error probability $\alpha$ is decreased from the level of 0.05 to an average level of 0.02 (for $h=1$, 2,3 ), whereas the coordinated Type II error probability $\beta$ displays a slight increase of about 0.01 from the average level of about 0.43 . For $h>h_{\alpha_{0}, \beta_{0}}^{*}$, the Type I error probability $\alpha$ is increased over the level of 0.05 , whereas the coordinated Type II error probability $\beta$ is decreased below 0.20 .

Ad option 2 (variance factor option (i))

The changes, being analogous to those in Option 1, are greater. For $h<h_{\alpha_{0}, \beta_{0}}^{*}$, the probability $\alpha$ is decreased to an average level of 0.01 , while the coordinated Type II error probability $\beta$ displays a slight increase of about 0.02 .

Based on the above results, it seems reasonable to apply modification only for $h<h_{\alpha_{0}, \beta_{0}}^{*}$, and thus for single point displacements. Either of the Options, as advantageous in terms of testing errors probabilities, is acceptable. Instead of the diversified level of $\alpha_{\mathrm{o}, \mathrm{m}}$ for different $h$, the averaged common values 0.02 and 0.01 can be recommended for Option 1 and Option 2, respectively. However, since it was found in computations that for $h=3$ in Option 2 (with $\left.\alpha_{\mathrm{o}, \mathrm{m}}=0.01\right) E_{\mathrm{sig}, \mathrm{m}}$ lies slightly outside $E_{\mathrm{sen}}$, the significance level $\alpha_{\mathrm{o}, \mathrm{m}}=0.015$ is proposed for Option 2. With this level $E_{\text {sig,m }}$ lies inside $E_{\text {sen }}$, i.e., $u_{\mathrm{o}, \mathrm{m}}<\lambda_{\mathrm{o}}$.

It was verified that the above conclusions also apply to the variance factor option (ii) the more, the greater the $r$. The computations showed that for $h=3$ in Option 2 with $\alpha_{\mathrm{o}, \mathrm{m}}=0.015 \hat{E}_{\text {sig,m }}$ lies inside $\hat{E}_{\mathrm{sen}}$ (i.e., $w_{\mathrm{o}, \mathrm{m}}<\lambda_{\mathrm{F}, \mathrm{o}}$ ) for $r \geq 23$. For $h=2$ and $h=1$, we have $r \geq 10$ and $r \geq 7$, respectively.
Table 5 Data for Option 1; variance factor option (i)

Table 6 Data for Option 2; variance factor option (i)

\begin{tabular}{rrrlllllllll}
\hline$h$ & $u_{\mathrm{o}, \mathrm{m}}$ & \multicolumn{1}{c}{$\lambda_{\mathrm{o}}$} & $\alpha_{\mathrm{o}, \mathrm{m}}$ & $\bar{\beta}_{m}$ & $\bar{\beta}$ & $h$ & $u_{\mathrm{o}, \mathrm{m}}$ & $\lambda_{\mathrm{o}}$ & $\alpha_{\mathrm{o}, \mathrm{m}}$ & $\bar{\beta}_{m}$ & $\bar{\beta}$ \\
\hline 1 & 5.85 & 7.85 & 0.016 & 0.500 & 0.500 & 10 & 17.27 & 16,24 & 0.069 & 0.138 & 0.145 \\
2 & 7.81 & 9.64 & 0.020 & 0.427 & 0.417 & 20 & 26.19 & 20,96 & 0.160 & 0.032 & 0.045 \\
3 & 9.36 & 10.90 & 0.025 & 0.370 & 0.358 & 30 & 34.16 & 24.55 & 0.274 & 0.007 & 0.014 \\
\hline
\end{tabular}

\begin{tabular}{rrllllllll}
\hline$h$ & $u_{\mathrm{o}, \mathrm{m}}$ & $\alpha_{\mathrm{o}, \mathrm{m}}$ & $\bar{\beta}_{m}$ & $\bar{\beta}$ & $h$ & $u_{\mathrm{o}, \mathrm{m}}$ & $\alpha_{\mathrm{o}, \mathrm{m}}$ & $\bar{\beta}_{m}$ & $\bar{\beta}$ \\
\hline 1 & 7.85 & 0.005 & 0.500 & 0.500 & 10 & 16.24 & 0.093 & 0.130 & 0.145 \\
2 & 9.64 & 0.008 & 0.435 & 0.417 & 20 & 20.96 & 0.399 & 0.019 & 0.045 \\
3 & 10.90 & 0.012 & 0.379 & 0.358 & 30 & 24.55 & 0.747 & 0.002 & 0.014 \\
\hline
\end{tabular}


Table 7 Data for Option 2, variance factor option (i), $\alpha_{\mathrm{o}, \mathrm{m}}=0.015$

\begin{tabular}{rlrrrrr}
\hline$h$ & $u_{\mathrm{o}}$ & \multicolumn{1}{c}{$u_{\mathrm{o}, \mathrm{m}}$} & \multicolumn{1}{c}{$\lambda_{\mathrm{o}}$} & $\alpha_{\mathrm{o}, \mathrm{m}}$ & $\bar{\beta}_{m}$ & $\bar{\beta}$ \\
\hline 1 & 3.84 & 5.92 & 7.85 & 0.015 & 0.500 & 0.500 \\
2 & 5.99 & 8.40 & 9.64 & 0.015 & 0.430 & 0.417 \\
3 & 7.81 & 10.46 & 10.90 & 0.015 & 0.377 & 0.358 \\
\hline
\end{tabular}

If the above-mentioned restrictions on the value of $r$ are acceptable, the significance level $\alpha_{\mathrm{o}, \mathrm{m}}=0.015$ can be proposed for both options (i) and (ii) in Option 2. Otherwise, the value of $\alpha_{\mathrm{o}, \mathrm{m}}$ for option (ii) should be increased accordingly. The data for variance factor option (i) are presented in Table 7.

A similar modification can be carried out for confidence area determination (variance factor option (i)). The modified confidence level $\gamma_{\mathrm{o}, \mathrm{m}}$ would be 0.98 for Option 1 and 0.985 for Option 2.

The choice of Options for both Task Cases I and II can be made depending on the type of practical applications.

The determined measures of MDD support and the proposed threshold modifications have a general character and are valid for any realization of Task Case I or Task Case II. Therefore, enclosing a numerical example did not seem to be necessary.

\section{Concluding remarks}

Theoretical findings of the research can be summarized as follows:

- determining Type II error probability $\bar{\beta}$ corresponding to Type I error probability $\alpha_{\mathrm{o}}$,

- introducing a rescaled Fisher-distributed test statistic which made it possible to construct a significance ellipsoid comparable with the sensitivity ellipsoid for the case when the a posteriori variance factor is used,

- proposing a 2-parameter measure $Q$ of MDD support in the accuracy analysis and significance test of displacements.

The computations show that the MDD support both in confidence region determination and significance test of single point displacements is the smallest for $1 \mathrm{D}$ networks. It increases successively for 2D and 3D networks. For global network displacements, with the increase in $h$, the MDD support increases with regard to $\beta$ much more than for single point displacements but decreases with regard to the second parameter in $Q$. Based on the analysis of MDD support, two options of modifying the significance and confidence thresholds for single point displacements are proposed. The modified thresholds being closer to the detectability threshold, correspond to significance level $\alpha=0.02$ or $\alpha=0.015$ and the confidence level $\gamma=0.98$ or $\gamma=0.985$. The advantage of these modifications is that the proposed decrease in the significance level $\alpha$ (or increase in confidence level $\gamma$ ) results in a negligibly small increase in the Type II error probability $\beta$.

In future research, it is planned to consider other types of displacement monitoring models and also, based on publications such as, e.g., (Xu et al. 2000) to examine the possibility of extending the findings of the present paper onto geometrical deformation approach.

\section{Appendix A}

\section{Another proof for datum invariance of sensitivity quadratic form}

Thesis A necessary and sufficient condition for datum invariance of the form $\Phi$ in a model (1) is that the coefficient matrix $\mathbf{S}$ in minimum-constraint datum definition eliminating the model defect is of the rank equal to that defect.

Proof Substituting $\hat{\mathbf{d}}$ and $\mathbf{C}_{\hat{\mathbf{d}}}$ as in (5) into the formula $\Phi=\hat{\mathbf{d}}^{\mathrm{T}} \mathbf{C}_{\hat{\mathbf{d}}}^{+} \hat{\mathbf{d}}$ (as in (7)), we get

$$
\begin{aligned}
\Phi & =(\Delta \mathbf{l})_{\mathrm{s}}^{\mathrm{T}} \mathbf{A}_{\mathrm{s}}\left(\mathbf{A}_{\mathrm{s}}^{\mathrm{T}} \mathbf{A}_{\mathrm{s}}\right)_{\mathrm{S}}^{-}\left[\left(\mathbf{A}_{\mathrm{s}}^{\mathrm{T}} \mathbf{A}_{\mathrm{s}}\right)_{\mathrm{S}}^{-}\right]^{+}\left(\mathbf{A}_{\mathrm{s}}^{\mathrm{T}} \mathbf{A}_{\mathrm{s}}\right)_{\mathrm{S}}^{-} \mathbf{A}_{\mathrm{s}}^{\mathrm{T}}(\Delta \mathbf{l})_{\mathrm{s}} \\
& =(\Delta \mathbf{l})_{\mathrm{s}}^{\mathrm{T}} \mathbf{A}_{\mathrm{s}}\left(\mathbf{A}_{\mathrm{s}}^{\mathrm{T}} \mathbf{A}_{\mathrm{s}}\right)_{\mathrm{S}}^{-} \mathbf{A}_{\mathrm{s}}^{\mathrm{T}}(\Delta \mathbf{l})_{\mathrm{s}}
\end{aligned}
$$

According to (Rao and Mitra 1971; Rao 1973), the expression $\mathbf{A}\left(\mathbf{A}^{\mathrm{T}} \mathbf{A}\right)^{-} \mathbf{A}^{\mathrm{T}}$, where $\left(\mathbf{A}^{\mathrm{T}} \mathbf{A}\right)^{-}$denotes a generalized inverse of $\mathbf{A}^{\mathrm{T}} \mathbf{A}$, does not depend on the choice of the generalized inverse.

Hence, for any given $(\Delta \mathbf{l})_{\mathrm{s}}$, excluding $(\Delta \mathbf{l})_{\mathrm{s}} \in \operatorname{Ker}\left(A_{\mathrm{s}}^{\mathrm{T}}\right)$, i.e., $\mathbf{A}_{\mathrm{s}}^{\mathrm{T}}(\Delta \mathbf{l})_{\mathrm{s}}=0$, the form $\Phi$ will have the same value for any type of the inverse $\left(\mathbf{A}_{\mathrm{s}}^{\mathrm{T}} \mathbf{A}_{\mathrm{s}}\right)_{\mathrm{S}}^{-}$used. This means any type of minimum-constraint datum definition [see (2)].

It follows immediately from the proof that for any overconstraint datum definition (i.e., where rank $\mathbf{S}>d$ ) the form $\Phi$ is not datum invariant. 


\section{Appendix B}

\section{Non-centrality parameter for the model (1) with weights of observation differences}

The displacement vector $\hat{\mathbf{d}}$ and its covariance matrix $\mathbf{C}_{\hat{\mathbf{d}}}$ (and $\hat{\mathbf{C}}_{\hat{\mathbf{d}}}$ ) are as follows

$$
\begin{aligned}
\hat{\mathbf{d}} & =\left(\mathbf{A}^{\mathrm{T}} \mathbf{P}_{\Delta \mathbf{l}} \mathbf{A}\right)_{\mathbf{S}}^{-} \mathbf{A}^{\mathrm{T}} \mathbf{P}_{\Delta \mathbf{l}} \Delta \mathbf{l} \\
\mathbf{C}_{\hat{\mathbf{d}}} & =\sigma_{\mathrm{o}, \mathrm{w}}^{2}\left(\mathbf{A}^{\mathrm{T}} \mathbf{P}_{\Delta \mathbf{l}} \mathbf{A}\right)_{\mathbf{S}}^{-} \\
\hat{\mathbf{C}}_{\hat{\mathbf{d}}} & =\hat{\sigma}_{\mathrm{o}, \mathrm{w}}^{2}\left(\mathbf{A}^{\mathrm{T}} \mathbf{P}_{\Delta \mathbf{l}} \mathbf{A}\right)_{\mathbf{S}}^{-}
\end{aligned}
$$

where $\mathbf{P}_{\Delta \mathrm{l}}$-weight matrix (pos. definite), $\sigma_{\mathrm{o} w \mathrm{w}}^{2}$-a priori variance of the observation difference of unit weight, $\hat{\sigma}_{\mathrm{o}, \mathrm{w}}^{2}$ —estimator (unbiased) of $\sigma_{\text {o,w }}^{2}$.

Denoting $\hat{\mathbf{C}}_{\hat{\mathbf{d}}}$ as $\hat{\mathbf{C}}_{\hat{\mathbf{d}}}=\hat{\sigma}_{\mathrm{o}, \mathrm{w}}^{2} \mathbf{G}$, where $\mathbf{G}=\left(\mathbf{A}^{\mathrm{T}} \mathbf{P}_{\Delta \mathbf{l}} \mathbf{A}\right)_{\mathbf{S}}^{-}$, the test statistic $\Psi$ corresponding to that in (18), will be

$\Psi=\frac{\hat{\mathbf{d}}^{\mathrm{T}} \mathbf{G}^{+} \hat{\mathbf{d}}}{\hat{\sigma}_{\mathrm{o}, \mathrm{w}}^{2}}$

Due to the property that $\Psi=\lambda_{\mathrm{F}}$ (as in Sect. 3.1, variance factor option (ii)), we get the non-centrality parameter for the analyzed option of the model (1).

We can check the same convergence of $\Psi$ to $\Phi$, i.e., since with $r \rightarrow \infty \hat{\sigma}_{\mathrm{o}, \mathrm{w}}^{2} \rightarrow \sigma_{\mathrm{o}, \mathrm{w}}^{2}$, so $\Psi \rightarrow \frac{\hat{\mathbf{d}}^{\mathrm{T}} \mathbf{G}^{+} \hat{\mathbf{d}}}{\sigma_{\mathrm{o}, \mathrm{w}}^{2}} \equiv \Phi$.

Acknowledgements The authors would like to thank Prof. Cüneyt Aydin of Yildiz Teknik University in Istanbul for sharing the program for computing non-centrality parameter for the $\chi^{2}$ and $F$ distributed test statistics. Thanks are also due to the anonymous Reviewers for their insightful and constructive remarks.

Author contributions W.P. theory, analyses and text; S.Ł. computation, tables and figures.

Data availability All data generated during the current study are available in this published article.

Open Access This article is licensed under a Creative Commons Attribution 4.0 International License, which permits use, sharing, adaptation, distribution and reproduction in any medium or format, as long as you give appropriate credit to the original author(s) and the source, provide a link to the Creative Commons licence, and indicate if changes were made. The images or other third party material in this article are included in the article's Creative Commons licence, unless indicated otherwise in a credit line to the material. If material is not included in the article's Creative Commons licence and your intended use is not permitted by statutory regulation or exceeds the permitted use, you will need to obtain permission directly from the copyright holder. To view a copy of this licence, visit http://creativecommons.org/licenses/by/4.0/.

\section{References}

Alizadeh-Khameneh MA, Eshagh M, Sjöberg LE (2015) Optimisation of Lilla Edet landslide GPS monitoring network. J Geod Sci 5:57-66. https://doi.org/10.1515/jogs-2015-0005

Aydin C (2011) Power of global test in deformation analysis. J Surv Eng 138:51-56. https://doi.org/10.1061/(ASCE)SU.1943-5428. 0000064

Aydin C, Demirel H (2005) Computation of Baarda's lower bound of the non-centrality parameter. J Geod 78:437-441. https://doi.org/ 10.1007/s00190-004-0406-1

Baarda W (1968) A testing procedure for use in geodetic network. Publications on Geodesy, New Series, Netherlands Geodetic Commission, Delft

Even-Tzur G (2002) GPS vector configuration design for monitoring deformation networks. J Geod 76:455-461. https://doi.org/10. 1007/s00190-002-0274-5

Even-Tzur G (2010) More on sensitivity of a geodetic monitoring network. J Appl Geod 4:55-59. https://doi.org/10.1515/jag.2010.006

Gaida W, Koch KR (1985) Solving the cumulative distribution function of the noncentral F-distribution for the noncentrality parameter. Sci Bull Stanisl Staszic Univ Min Metall Geod b:35-43

Heck B (1986) Sensivitätsanalyse geodätischer Deformationsnetze. Allg Vermessungs-Nachrichten 169-181

Hsu R, Hsiao K (2002) Pre-computing the sensitivity of a GPS station for crustal deformation monitoring. J Chin Inst Eng 25:715-722. https://doi.org/10.1080/02533839.2002.9670745

Kuang S (1991) Optimization and design of deformation monitoring schemes. Ph.D. dissertation. University of New Brunswick, Department of Surveying Engineering

Niemeier W (1982) Principal component analysis and geodetic networks - some basic considerations. Proc Surv Control Netw Heft 7:275-291

Niemeier W (1985) Anlage von Überwachungsnetzen. In: Pelzer H (ed) Geodaetische netze in landes-und ingenieurvermessung II. Verlag Konrad Wittwer, Stuttgart, pp 527-558

Niemeier W, Teskey WF, Lyall RG (1982) Precision, reliability and sensitivity aspects of an open pit monitoring network. Aust J Geod Photogramm Surv 37:1-27

Pelzer H (1972) Nachweis von Staumauerdeformationen unter Anwendung statistischer Verfahren. Berichte Arbeitsgruppe B, Deutscher Geodätentag Braunschweig

Pope SB (2008) Algorithms for ellipsoids. Sibley School of Mechanical and Aerospace Engineering, Cornell University 14853, Report FDA-08-01

Rao CR, Mitra S (1971) Generalized inverse of matrices and its applications. Wiley, New York

Rao CR (1973) Linear statistical inference and its applications, 2nd edn. Wiley, New York, ISBN 0-471-70823-2

$\mathrm{Xu}$ PL (1995) Testing the hypotheses of non-estimable functions in free net adjustment models. Manuscr Geod 20:73-81

Xu PL (1997) A general solution in geodetic nonlinear rank-defect models. Boll Geod Sci Aff 56:1-25

$\mathrm{Xu}$ PL et al (2000) Invariant geodynamical information in geometric geodetic measurements. Geophys J Int 142:586-602

Yazji S (1997) The optimal design of the monitoring network of a ground fault in Syria. Acta Geod Geophys Hung 32:87-103. https://doi.org/10.1007/BF03325480 